\title{
Comment concevoir des référentiels de diplômes professionnels sans renoncer au travail réel ?
}

How to design standard frame of reference of vocational diploma without giving up the real work?

\section{Stéphane Balas}

\section{(2) OpenEdition}

\section{Journals}

Édition électronique

URL : http://journals.openedition.org/activites/2889

DOI : 10.4000/activites.2889

ISSN : $1765-2723$

Éditeur

ARPACT - Association Recherches et Pratiques sur les ACTivités

\section{Référence électronique}

Stéphane Balas, «Comment concevoir des référentiels de diplômes professionnels sans renoncer au travail réel ? », Activités [En ligne], 13-2 | 2016, mis en ligne le 15 octobre 2016, consulté le 19 avril 2019. URL : http://journals.openedition.org/activites/2889 ; DOI : 10.4000/activites.2889

Ce document a été généré automatiquement le 19 avril 2019

\section{(i)

Activités est mis à disposition selon les termes de la licence Creative Commons Attribution - Pas d'Utilisation Commerciale - Pas de Modification 4.0 International. 


\section{Comment concevoir des référentiels de diplômes professionnels sans renoncer au travail réel ?}

How to design standard frame of reference of vocational diploma without giving up the real work?

Stéphane Balas

\section{NOTE DE L'ÉDITEUR}

Article soumis le 24 novembre 2015, accepté le 3 mars 2016

\section{Introduction}

Disons-le d'emblée : l'activité de travail, c'est-à-dire la part que l'homme apporte de lui, face à la prescription (Leplat, 2002), ne peut s'extraire comme une substance et être formalisée, comme telle, dans un référentiel. Même si certains discours managériaux peuvent alimenter cette illusion, aucun spécialiste de l'analyse de l'activité en vue de la formation (Champy-Remoussenard, 2005) n'adhère à ce rapport linéaire entre une activité et sa formalisation descriptive.

2 L'activité de travail est complexe, dynamique, labile et toute tentative de formalisation dans un document textuel, par exemple au moment de la rédaction d'un référentiel, entraîne aussi en partie sa dénaturation. "L'activité » décrite dans un référentiel de diplôme professionnel n'est pas pleinement une activité mais plus souvent une tâche (plus ou moins précisément explicitée) (Maillard, 2001) ou encore une trace du comportement moyen observé (ce qui correspondrait à une "action-type ») ou des éléments de contexte relevant plutôt de l'organisation du travail (par exemple les fonctions). 
3 Dans tous les cas, le référentiel se fait porteur de «ce qui est à faire » plutôt que de «ce qui se fait»; il traduit plutôt ce qui relève de la prescription, et encore en partie seulement ${ }^{1}$, sans jamais parvenir à s'approcher du réel.

Cependant, cette distance entre le travail réel et un outil prescriptif, si elle apparait naturelle au premier abord puisque par définition la prescription n'est pas l'activité réalisée, pose problème. Soigner sa prescription, c'est au contraire la faire reposer sur une connaissance précise du réel et instaurer un rapport itératif entre les différentes instances du métier (Clot, 2008a), en particulier les instances impersonnelles (la prescription) et transpersonnelles (le genre du métier). Sans ce rapport, sans cette volonté systématique de prise en compte du réel, la prescription, et ici les outils de cette prescription, risque fort d'être dénuée de sens, floue et perçue comme artificielle, c'est-àdire pour les usagers comme des instruments de contrôle et non d'orientation de l'action. C'est par exemple le constat fait par les formateurs portugais face au référentiel de compétence clé pour la mise en œuvre d'une validation des acquis (Fernandes \& Santos, 2014) qui les obligent à se confronter, seuls, aux contradictions de ce processus certificatif.

5 Partant de ce constat, nous allons, dans ce texte, observer ce phénomène social actuel d'inflation de référentiels dans le champ de la formation professionnelle, mais aussi dans l'entreprise et au-delà ${ }^{2}$ et nous interroger plus particulièrement sur l'activité de conception de ces référentiels. En effet, si quelques travaux s'intéressent aux usages de ces référentiels dans l'entreprise (Ughetto, 2014), en formation (Boccara, Vidal-Gomel, Rogalski, \& Delhomme, 2014; Huchette, 2014) ou encore pour la certification (Maillard, 2007), peu se concentrent sur les processus de fabrication des référentiels de diplômes professionnels, entre travail et formation, à l'exception notable de quelques travaux sur la validation des acquis de l'expérience (Mayen \& Métral, 2008; Prot, 2003) et les importants écrits de Figari (1994) sur la fonction évaluative de ces référentiels.

6 Après être revenu, dans un premier temps, sur ce processus de conception de référentiels analysé comme une activité (Olry \& Vidal-Gomel, 2011), nous chercherons à montrer que le travail est l'objet d'une triple euphémisation lors de la tentative de produire un référentiel descriptif de diplôme : euphémisation liée à une analyse au mieux partielle de ce travail, euphémisation en rapport au dialogue social qui se noue autour de la production de ce référentiel et brouille les frontières entre travail et emploi (Jobert, 2013) ou encore euphémisation due à l'intégration du diplôme produit dans un système éducatif préexistant, impliquant des contraintes formelles fortes. Dès lors, le produit final est souvent très éloigné du travail réel.

7 Précisons que l'observation de cette triple euphémisation est un constat, non un jugement car comme dans tout système sociotechnique (Akrich, 1991) complexe, il faut mesurer chaque fait à l'aune de ses répercussions sur les autres éléments du système. Par exemple, l'existence de contraintes liées au dialogue social permet, par ailleurs, de garantir la légitimité du référentiel produit dans le secteur visé car ce référentiel constitue alors un support de « régulation sociale normative » (Chauvigné, 2010).

8 Dans un deuxième temps, nous présenterons le cadre conceptuel que nous mobilisons pour analyser ces processus. Inscrit dans une approche clinique du travail, nous considérons qu'il est nécessaire d'analyser cette conception avec un regard systémique, développemental et instrumenté (Albero \& Brassac, 2013), c'est-à-dire, comme nous le verrons, observer un phénomène en le considérant comme un système comprenant 
divers éléments en interaction mais aussi chercher, dans une tradition vygotskienne, à identifier les ressorts de développement passés ou futurs de ce système. Enfin, nous portons une attention particulière aux instruments mobilisés par les acteurs dans ce système car ils nous semblent particulièrement révélateurs de leur activité.

En particulier, nous attacherons une grande place aux "produits» de ces activités de conception, à partir d'une analyse de référentiels. La méthode mobilisée est donc une approche documentaire, mais en considérant les constats issus du travail documentaire comme les traces laissées par les conflits d'activités vécus (Leplat \& Cuny, 1974) par les concepteurs lors de la conduite des référentialisations (Figari, 1994, op.cit.) entre "référés et référents ». Ainsi, notre méthode est indirecte (Vygotski, 1925/2005) : nous nous concentrons sur les documents produits, non pour eux-mêmes comme dans une analyse méthodologique, mais pour approcher l'activité des concepteurs.

Nous poursuivrons ce travail par un exposé de quelques-uns de ces constats, à partir de l'analyse de quelques référentiels, propres à éclairer notre remarque initiale sur l'écart entre référentiel et réel de l'activité (Clot, 2004) à partir de l'activité des concepteurs de diplômes professionnels. Ces constats seront finalement discutés, pointant en particulier les limites de cette démonstration et indiquant des perspectives à ce travail. Nous conclurons enfin cet article en rappelant l'importance de penser «travail» quand on souhaite produire des référentiels.

\section{Problématique}

11 Si l'on constate aujourd'hui une multiplication des référentiels qui tentent de répondre à des enjeux de rationalisation et de développement de l'efficience de l'action humaine face à des processus sociaux de plus en plus complexes (organisation du travail, dispositif de formation, progression pédagogique scolaire), on peut observer plusieurs types de réaction.

Dans certains cas, les référentiels sont promus comme des « solutions » qui se substituent au travail d'organisation du travail dans l'entreprise, assumé normalement par les manageurs et la direction. Le référentiel, d'outil complémentaire participant à la définition de la prescription du travail de chacun, devient la norme intangible par rapport à laquelle on mesure la conformité du travail des individus. On retrouve ici une des dérives des référentiels de compétences, dont la fonction est plus évaluative que descriptive (Coulet, 2011).

À l'opposé, certains dénoncent les référentiels comme des avatars d'une forme de rationalisation de l'activité humaine, insupportable car porteuse de schémas dépassés (quant au mode d'encadrement plus directif que participatif) et privant les individus de possibilités d'innovation ou de développement de leur créativité. Dans le secteur de la formation professionnelle pour adultes, on dénonce par exemple les «formations prescriptives", entendu qu'un dispositif organisé par des référentiels sera obligatoirement fondé sur une pédagogie descendante, non-inductive. Dans ce mode de raisonnement, la prescription est comprise, par exemple dans les approches ergologiques, comme une forme "d'hétérodétermination » (Schwartz, 2007, p. 130) à laquelle il faut résister pour agir et être. Selon cette approche, en effet, la prescription est assimilée à une contrainte externe, l'homme agissant sur le monde en s'en émancipant et se faisant, en se construisant lui-même comme acteur autonome. 

un rejet de cette norme. N'est-il pas possible d'échapper à cette alternative et de proposer, face aux référentiels, une «troisième voie » (Clot, 2000 ; Maggi, 2011), plus développementale?

lesparaît, comme nous l'avons indiqué, que les travaux portant sur le " phénomène » référentiel, s'intéressent plus à l'usage de ces référentiels, aux apports et aux limites de ces derniers et ne reviennent que très peu à la racine de la question, c'est-à-dire au processus de leur conception. En effet, celui-ci est doublement essentiel pour comprendre les référentiels et observer les rapports qu'ils entretiennent avec le travail réel : revenir à la conception permet de lever une partie du voile sur les objectifs qui animent les concepteurs, de comprendre leur propre représentation de leur rôle de concepteur mais aussi de la place qu'ils souhaitent accorder à une description des activités réelles de travail du métier visé par le référentiel et dont ils sont des experts. De plus, on connait le lien entre conception d'un dispositif et son usage, ce dernier étant anticipé lors de la fabrication (Akrich, 1991, op.cit.) au point de devoir distinguer le dispositif idéel puis fonctionnel du dispositif vécu (Albero, 2010). Analyser la conception permet d'embrasser l'ensemble du phénomène et surtout de distinguer cause (dans la conception) et conséquence (dans l'usage).

16 Cependant, les travaux portant sur la conception des référentiels sont souvent à caractère méthodologique (Jouvenot \& Parlier, 2005). De manière souvent très complète, ces travaux cherchent à guider les concepteurs dans leur rédaction en vue de produire des référentiels les plus clairs, précis et logiques. Deux éléments, cependant, nous apparaissent notables. D'une part ces travaux s'intéressent particulièrement à la compétence et aux référentiels de compétences en faisant l'impasse sur une phase, qui nous paraît préalable, de définition d'un référentiel descriptif (Balas, 2011a) à partir de l'activité. D'autre part et assez logiquement avec ce qui précède, le grand absent de ces guides est le travail entendu comme la part créative de l'homme, en réponse à la prescription.

17 Certaines approches se déclarent plus proches d'une véritable analyse de ce travail, en rapport avec la conception de référentiels de diplômes professionnels. On classe ici les travaux initiés par Nicole Mandon (2009) au Céreq et sa méthode ETED, pour «EmploiType Etudié dans sa Dynamique » (Liaroutzos \& Sultzer, 2006) qui est un outil d'analyse des emplois à partir du travail et de son exercice concret. Cette méthode, très utilisée dans l'entreprise comme en formation professionnelle, propose à partir du recueil des propos des "titulaires d'emploi» et de leur hiérarchie, de répertorier les situations professionnelles regroupées en fonction de leur proximité. Sont alors construits des emplois-types.

«Ce processus d'identification de sorte "d'idéal-type", d'influence wébérienne, permet à la méthode ETED d'inspirer des ingénieries de certification » (Balas, 2011a, op. cit.).

$\mathrm{Au}$ final, ce qui est référentialisable dans le réel du travail, c'est l'emploi-type, ce qui inspire quelques critiques. D'abord, la vision du travail adoptée par cette méthode oscille entre celui «à faire », défini par l'encadrement du salarié et l'organisation du travail, et celui « qui se fait », obtenu par un recueil direct auprès du professionnel. Cependant, la critique la plus vive que nous pouvons adresser à cette conception est liée à la méthode d'analyse du «travail réel » exclusivement tournée vers le recueil du déclaratif. Mais « la narration en tant que telle prend déjà part à ce processus réflexif à travers la sélection et 
la mise en lien - "la mise en intrigue", disait Ricœur - des éléments qui sont relatés " (Lainé, 2011, p. 87). Dire son travail, c'est déjà le reconstruire. Prendre le déclaratif pour «argent comptant» est simplificateur de la réalité du travail. On dispose alors d'une vision appauvrie, reconstruite a posteriori du travail réel.

Analyser la conception des référentiels à partir d'une analyse indirecte des traces des activités de conception impose donc de s'inscrire dans un cadre conceptuel où sont pensés les rapports entre conception et usage. C'est ce que nous allons développer maintenant.

\section{De la clinique de l'activité à la praxéologie de la connaissance}

\subsection{Cadre conceptuel}

19 À l'origine inscrites dans le cadre de la «clinique de l'activité » (Clot, 1999) au service d'objets relevant du champ des sciences de l'éducation, nos pratiques nous amènent, au contact des obstacles empiriques et d'une volonté de transdisciplinarité, à enrichir notre approche. Aujourd'hui, sans renier les apports fondateurs de cette clinique, nous adoptons, en vue d'instruire un dialogue plus large avec les sciences de l'éducation mais aussi les sciences $\mathrm{du}$ travail, quelques principes théoriques et méthodologiques complémentaires.

20 Le premier est de considérer les objets traités (activités, compétences, transmission, dispositifs dont les référentiels) du point de vue de leur développement, en particulier au niveau de leur signification pour les acteurs, signification qui constitue "l'unité de base " qui possède «les propriétés du tout [...] sous la forme initiale la plus simple » (Vygotski, 1934/1997, p. 417) et qui permet de saisir les mobiles des actions des individus, en particulier ici des concepteurs. Cette signification est aussi un «outil socialement fabriqué en fonction de tâches données » (Vygotski, 2014, p. 47), un médiateur artificiel (culturel) de l'activité.

21 Le deuxième est de porter sur ces individus et les organisations (de travail, d'éducation) un regard systémique. Un dispositif de formation, par exemple, peut être vu comme un «système fonctionnel» que Hoc (1996) définit comme un «système comprenant des individus et les artefacts avec lesquels ils interagissent ».

22 Dès lors, le troisième principe est de porter une attention particulière aux « objets, artefacts, outils et instruments, qu'ils soient matériels, virtuels ou symboliques » (Albero \& Brassac, 2013, op. cit., p. 110) et de les considérer comme des indices précieux du « réel de l'activité » (Clot, 2008a) des acteurs.

Cette triple posture correspond assez précisément à ce qu'Albero et Brassac (2013, op. cit.) définissent comme une "praxéologie de la connaissance » qui pourrait constituer une première piste "vers un construit transdisciplinaire adapté » (ibid., p. 113) aux enjeux éducatifs actuels. En effet, selon ces auteurs, les questions éducatives sont abordées au plan scientifique avec des points de vue atomisés ce qui nuit à l'efficacité de ces recherches. Observer les grandes questions éducatives (apprentissage, évaluation, didactique, pédagogie...) à partir de l'activité des acteurs et en considérant cette activité du point de vue de son inscription dans un système mais aussi à partir de son 
développement et de son "équipement instrumental» constitue donc une réponse syncrétique.

Nous nous inscrivons donc dans cette voie. Analyser un phénomène à partir de l'activité des acteurs concernés, ici les concepteurs de référentiels et considérer que cette activité, dont nous étudions le développement et ses invariants (Clot 2008b), s'inscrit dans un système dont on ne peut isoler les éléments constitutifs. Enfin, nous postulons que ces activités, dans l'épaisseur des contradictions vécues, laissent des traces dans les outils produits (ici les référentiels) que nous pouvons alors étudier en vue de «remonter » à l'activité. Précisons que ces contradictions sont consubstantielles de toutes activités puisque le réel est toujours en partie imprédictible. Ce que nous cherchons à saisir, à travers ces traces, ce sont les contradictions en tant qu'elles «trahissent» l'activité et non en tant que phénomène à réduire.

Comment étudier ces traces?

\subsection{L'analyse de l'activité comme méthodologie}

Nous avons réuni des référentiels de diplômes professionnels, de niveaux différents, provenant de ministères certificateurs variés et dont les méthodologies de conception sont distinctes. Ces documents sont globalement accessibles sur internet, pas toujours dans leur intégralité ; mais on peut compléter cette recherche par la lecture de la fiche du diplôme déposée au répertoire national des certifications professionnelles (cncp.gouv.fr). Cette fiche est un condensé de la rédaction complète du diplôme qui permet d'en saisir des éléments clés comme les modes d'évaluation ou encore les publics visés.

Nous appuyant sur une familiarité avec les référentiels et leur conception, puisque nous avons contribué à la rédaction de plusieurs d'entre eux et que nous animons des enseignements universitaires sur le thème des référentiels et de l'analyse de l'activité, nous avons sélectionné, parmi les très nombreux disponibles, quatre référentiels qui nous apparaissaient comme typiques des contradictions que nous souhaitions exposer. Ainsi, nous réalisons, dans un premier temps, une lecture globale des documents. Elle nous offre une compréhension de l'objet social du diplôme, parfois plus complexe à saisir qu'on ne le croit $^{3}$.

28 À partir de cette première lecture, nous passons à une seconde lecture, plus analytique, visant à détecter les traces des contradictions vécues par les concepteurs dans l'élaboration des référentiels et leurs articulations. Dans cette enquête documentaire, on «traque » les contradictions rédactionnelles, les euphémisations les plus évidentes qui peuvent masquer l'embarras des rédacteurs, l'usage de termes qui paraissent « déplacés » dans le référentiel, les répétitions...

Par exemple, certaines rédactions présentent le métier visé comme une succession d'étapes non reliées entre elles (diagnostique, intervention, vérification... comme dans le baccalauréat professionnel "électrotechnique, énergie, équipements communicants » Balas, Paddeu et Veneau, 2016). D'autres mobilisent pour décrire les compétences attestées par la certification la série de termes «Savoirs, savoir-faire techniques, savoirfaire relationnels, savoir-faire organisationnels» (Référentiel d'emploi Activités Compétences du titre professionnel d'Assistant(e) Ressources Humaines délivré par le ministère du travail, p. 16) qui constitue une atomisation artificielle des composantes de la compétence, par principe réunies. D’autres utilisent pour décrire des activités 
caractéristiques du métier visé des formules abscondes comme «Exploiter les données de la conception et de la réalisation" (Référentiel d'activités professionnelles du baccalauréat professionnel « Métiers de la mode - Vêtements », p. 21).

On observe aussi les articulations entre les différents référentiels : rapports de déduction, de causalité, de linéarité, d'inclusion... et on tente de détecter les liens qui apparaissent peu logiques ou « trop logiques » (voir l'exemple ci-dessous du référentiel de la profession de masseur-kinésithérapeute).

31 Ce travail permet de mettre en lumière quelques cas, exemplaires des contradictions vécues des concepteurs. Précisons que ces constats ne relèvent pas d'une évaluation externe sur la qualité des référentiels dont nous devrions alors étudier l'usage, ce qui n'est pas le cas ici.

\section{Quelques constats à partir de l'étude de référentiels}

Nos différentes analyses confirment la complexité de l'activité de conception des référentiels. Les concepteurs doivent gérer des conflits de valeurs multiples (Leplat \& Cuny, 1974, op. cit.). En effet, ces acteurs sont, dans la réalisation de ce travail, au cœur d'une «tourmente» que l'on peut caractériser par l'identification de diverses contradictions que ces professionnels doivent " gérer » dans l'action, sans jamais pouvoir les «régler» (Balas, 2011b). Notons que ces contradictions sont intrinsèques (liées à la nature même de l'activité de conception) mais aussi extrinsèques (quand ce sont les contradictions non réglées des prescripteurs ${ }^{4}$ qui, en cascade, impactent l'activité des concepteurs).

Les référentiels retenus pour cet article ont été sélectionnés à partir de leur diversité de niveaux (V, III, II), de champs professionnels (santé, sport, management) et d'origines (différents ministères, ordre professionnel).

\subsection{Le référentiel du CAP aéronautique : un usage pragmatique des concepts}

Parmi ces prescripteurs, l'Éducation Nationale fournit aux rédacteurs de référentiels (participants aux "groupes de travail») un "guide d'élaboration des diplômes professionnels" (2004) qui est un recueil de consignes méthodologiques visant en particulier à prescrire l'usage de nombreux concepts. Les termes de fonctions, tâches, activités et opérations (p.13) doivent être mobilisés pour rédiger le Référentiel d'Activité professionnelle (RAP), ainsi que celui de compétences pour le référentiel de certification. Cependant, selon Maillard (2003, p. 67), ce « guide méthodologique (est) en fait très peu prescripteur ».

On l'observe en étudiant le référentiel du CAP aéronautique. Ce diplôme, récemment rénové (arrêté publié en août 2013), propose, dès le début de son RAP, une précision importante (Figure $1^{5}$ ): 
Figure 1 : Extrait du référentiel d'activités professionnelles du CAP « aéronautique » (p. 3).

Figure 1: Extract from the frame of reference for professional activities within the "aeronautical" CAP ( $p$. 3)

\section{ACTIVITES ET TÂCHES PROFESSIONNELLES}

Les activités professionnelles décrites ci-après, déclinées à partir des fonctions d'entreprise, constituent le référentiel des activités professionnelles du titulaire du certificat d'aptitude professionnelle aéronautique. Les activités sont ensuite déclinées en tâches professionnelles et éventuellement en opérations selon le schéma général ci-dessous.

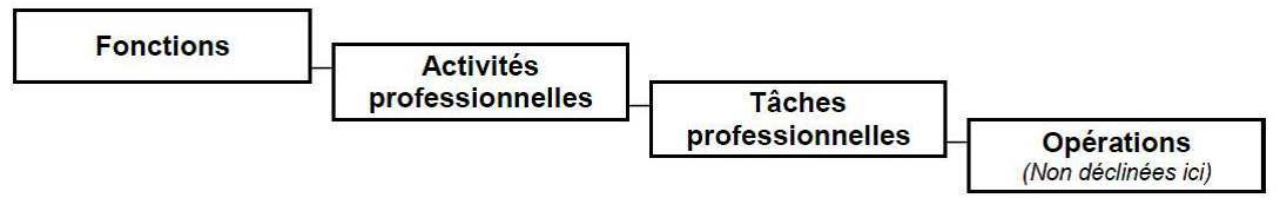

Si les rédacteurs mobilisent pour ce CAP les concepts de fonctions, activités professionnelles et tâches professionnelles, ils renoncent à celui d'opérations.

Dans bien des cas, entre la polysémie des termes et leurs rapports plus ou moins clairs (par exemple, y a-t-il un lien entre une fonction et des tâches?), les concepteurs de référentiels oscillent entre une recherche de rigueur dans l'usage de ces termes, ce qui ne favorise pas leur recherche de description authentique. Mais ils doivent aussi parfois en faire un usage pragmatique, par exemple en abandonnant la catégorie qui encombre, en redéfinissant un des termes (par exemple la tâche est une " petite activité ») ou encore en subdivisant une catégorie en ajoutant aux concepts des qualificatifs (une compétence " générale », ou une fonction " transversale »).

Ici, l'abandon du terme d'opération, d'autant plus surprenant au niveau d'un CAP qui vise à former des "opérateurs", s'accompagne d'un mode rédactionnel des activités et des tâches que l'on retrouve fréquemment dans les diplômes professionnels des secteurs industriels (Figure 2) : 
Figure 2 : Extrait du Référentiel d'Activités Professionnelles du CAP « aéronautique » (p. 5). Figure 2: Extract from the frame of reference for professional activities within the "aeronautical" CAP ( $p$.

5)

\begin{tabular}{|c|c|c|c|}
\hline & OPTION AVIONIQUE & OPTION SYSTEMES & OPTION STRUCTURE \\
\hline \multicolumn{4}{|c|}{ Activité 1 - PRÉPARATION DE L'OPERATION } \\
\hline \multicolumn{4}{|c|}{ 1. Description des tâches : } \\
\hline \multicolumn{4}{|c|}{ T1.1. Utiliser la documentation technique, pouvant être rédigée en anglais, spécifique à son opération. } \\
\hline T1.2. & $\begin{array}{l}\text { Vérifier la prèsence, la référ } \\
\text { des moyens (outillages, app }\end{array}$ & $\begin{array}{l}\text { des ensembles, sous-ensembl } \\
\text { ure...). }\end{array}$ & omposants, kits, consommables et \\
\hline T1.3. & Configurer l'environnement & travail en vue de l'opération. & \\
\hline
\end{tabular}

2. Résultats attendus :

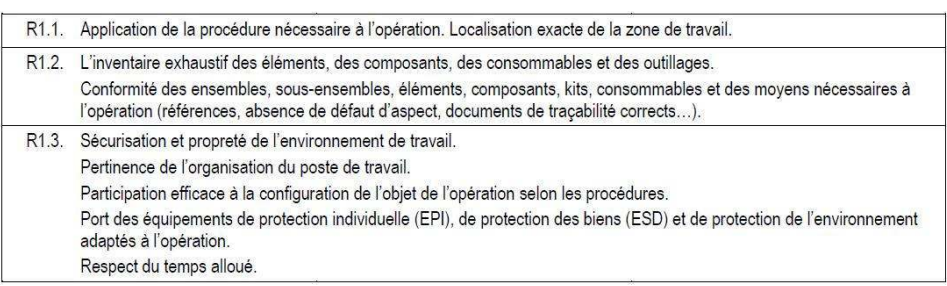

3. Conditions de réalisation :

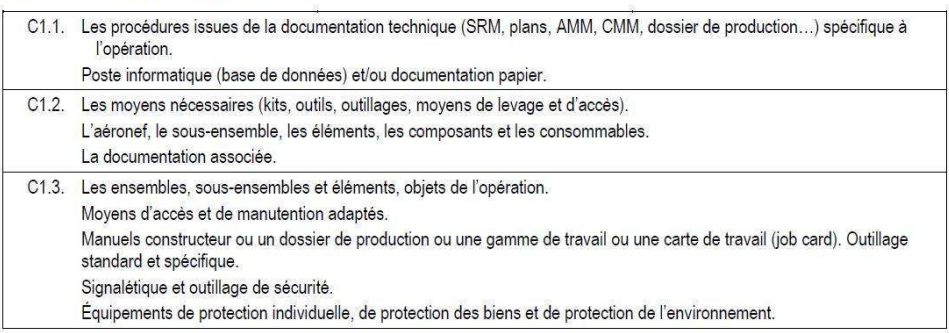

L'activité, en « chapeau » de la fiche, se décline en tâches (T1, T2...). Sont aussi précisés les résultats attendus (R1, R2...) ainsi que les conditions de réalisation $(\mathrm{C} 1, \mathrm{C} 2 \ldots)$.

Ce luxe de détails, sans nul doute très utile pour la mise en œuvre du référentiel (formation comme évaluation), renforce cependant le sentiment d'un usage restrictif des concepts. La proposition, à travers le guide, d'instituer un rapport d'emboitement (type « poupée russe ») entre les concepts de fonction, d'activité, de tâche et d'opération, n'aide sans doute pas les rédacteurs. En effet, si la fonction relève de l'organisation du travail (du côté de la direction et des manageurs, donc), les notions d'activités et de tâches sont, elles, dans des rapports bien différents si l'on se réfère aux apports de l'ergonomie francophone depuis le milieu du siècle dernier. Pour cette approche, l'activité est ce que l'homme développe pour répliquer à la tâche, ce qu'il apporte d'engagement subjectif face à une prescription, par nature, simplificatrice (Leplat \& Hoc, 1983; Poussin, 2010). L'opération, quant à elle, relève plus des moyens mis en œuvre pour être efficace, voire efficient (c'est-à-dire économe de ses moyens) dans l'atteinte du but concret de l'action (Leontiev, 1984). Cette description risque cependant de produire des unités de faible taille, mais également dépourvues de sens.

Comment alors proposer un cadre méthodologique moins complexe au plan sémantique et intégrant plus justement les différentes composantes du travail ? Comment mieux équiper les concepteurs de référentiels dans leur tentative de mieux prendre en compte les dimensions prescrites comme effective de l'activité humaine? 


\subsection{Le référentiel de la profession de masseur-kinésithérapeute : produire une norme ou inviter les usagers à produire leur norme?}

Le deuxième exemple est basé sur un référentiel concernant la profession de masseurkinésithérapeute. Contrairement au précédent, il ne s'agit pas d'un référentiel de diplôme ; il renvoie au souhait des rédacteurs qu'il serve de référence pour la réingénierie du Diplôme d'État, annoncée depuis plusieurs années par le Ministère de la Santé (Balas, 2014). De nombreuses réflexions sont menées depuis plusieurs années dans ce secteur, et ont conduit à l'adoption par l'ordre des masseurs-kinésithérapeutes d'un « référentiel de la profession » en 2013. Produit par des universitaires et des experts du métier, ce référentiel se présente sous la forme d'un document de 218 pages (dont 128 d'annexes). Le document principal comprend différentes parties, comme l'indique l'extrait ci-joint (Figure 3) :

Figure 3 : Extrait du référentiel de la profession de l'ordre des masseurs-kinésithérapeutes. Figure 3: Extract from the frame of reference of the profession of the order of physiotherapists

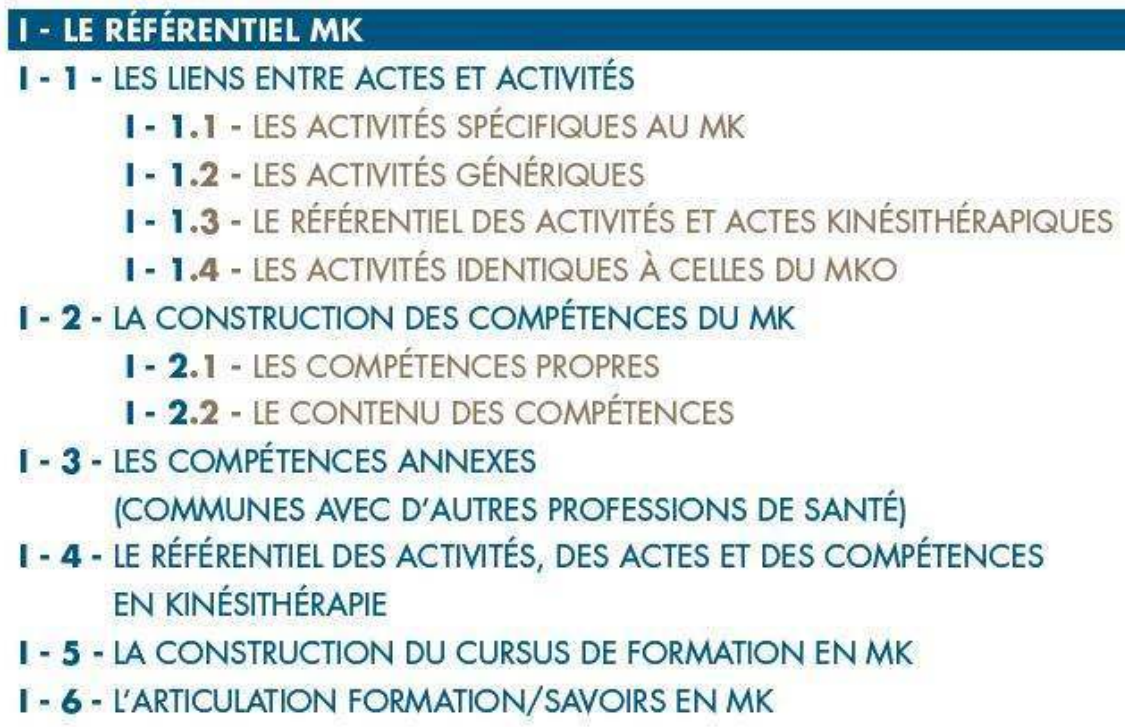

43 Cet exemple nous semble illustratif d'une contradiction consubstantielle du processus de référentialisation. « Normer ou renormaliser » : la question est de savoir si le référentiel a pour fonction de normer les pratiques des pédagogues (considérés alors comme des opérateurs) mais aussi des formés dans leur travail futur, ou, au contraire, de constituer une invitation à la renormalisation (Canguilhem, 2002) pour des " usagers acteurs », qui pourront s'emparer du référentiel pour en faire un instrument de développement de leurs pratiques. Selon le choix retenu, la manière de rédiger le référentiel sera bien différente. Dans un premier cas, une vaine tentation d'être exhaustif sera première alors que dans le second, c'est surtout le sens des activités, les motifs d'agir qui seront valorisés (Leontiev, 1984, op. cit.).

Ce document «porte la trace » de ces objectifs contradictoires qu'ont souhaité (ou dû ?) poursuivre les rédacteurs. Si la longueur du document semble créditer la thèse d'une recherche d'exhaustivité, de même que le traitement des questions de formation ${ }^{6}$, le soin 
apporté aux analyses historiques, juridiques et professionnelles invite plutôt le lecteur à considérer ce document comme un instrument de sa réflexion.

Cependant, une analyse plus précise du contenu du référentiel d'activité (partie 1.1 : les liens entre actes et activités) et de celui de compétences (partie 1.2: la construction des compétences $d u \mathrm{MK}$ ) laisse supposer que le souhait de normer les pratiques l'a emporté. Un indice de cette déduction est la proximité des intitulés des activités (six sont identifiées) et des compétences « propres » (cinq).

Les figures 4 et 5 ci-dessous reprennent les intitulés de deux activités (Établir un diagnostic kinésithérapique et Décider de la stratégie thérapeutique et des soins kinésithérapiques à mettre en cuvre) et des cinq "compétences propres", dont la C2. Élaborer un diagnostic kinésithérapique et la C3. Concevoir et conduire une stratégie d'intervention en kinésithérapie).

Figure 4 : Extrait de la page 43 du référentiel de la profession de l'ordre des masseurskinésithérapeutes.

Figure 4: Extract from page 43 of the frame of reference of the profession of the order of physiotherapists

\begin{tabular}{|c|c|}
\hline $\begin{array}{c}\text { Établir un diagnostic } \\
\text { kinésithérapique }\end{array}$ & $\begin{array}{l}\text { Identifier les risques même en situation d'urgence. } \\
\text { Analyser, synthétiser, problématiser, reconnaître les patholo- } \\
\text { gies et les situations qui impliquent l'exclusion du patient du } \\
\text { champ d'intervention kinésithérapique. } \\
\text { Établir un diagnostic intégrant un diagnostic d'exclusion et } \\
\text { de contre-indication. } \\
\text { Informer - Expliquer - Communiquer. }\end{array}$ \\
\hline $\begin{array}{l}\text { Décider de la } \\
\text { stratégie thérapeu- } \\
\text { tique et des soins } \\
\text { kinésithérapiques } \\
\text { à mettre en œuvre. }\end{array}$ & $\begin{array}{l}\text { Mettre en place une stratégie thérapeutique kinésithéra- } \\
\text { pique. } \\
\text { Choisir les orientations et les techniques kinésithérapiques. } \\
\text { Rechercher l'efficience pratique selon les données actuelles } \\
\text { de la science. } \\
\text { Planifier les soins kinésithérapiques. } \\
\text { Évaluer le bien fondé, l'efficacité et l'efficience des soins } \\
\text { prodigués. }\end{array}$ \\
\hline
\end{tabular}

Figure 5 : Extrait de la page 44 du référentiel de la profession de l'ordre des masseurskinésithérapeutes.

Figure 5: Extract from page 44 of the frame of reference of the profession of the order of physiotherapists

\section{I - 2 - LA CONSTRUCTION DES COMPÉTENCES DU MK}

\section{I - 2.1 - LES COMPÉTENCES PROPRES}

En lien avec la description des activités et des compétences, une liste de compétences génériques au $M K$ peut être rédigée :

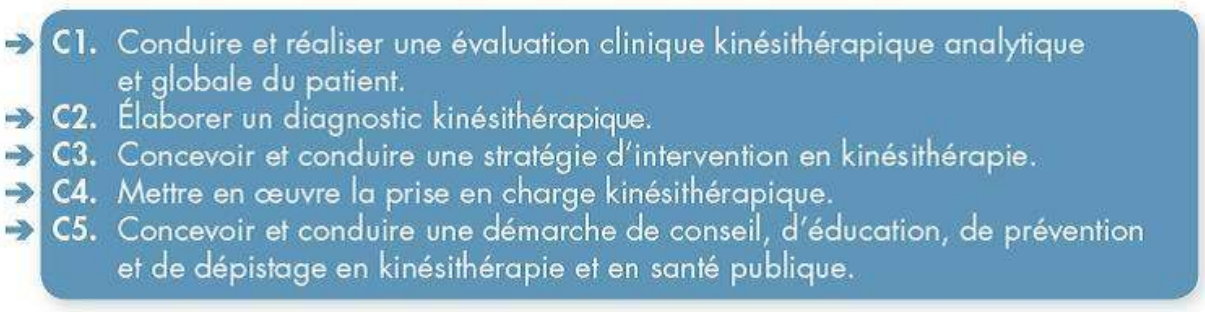



paraphrase de l'activité. Ce constat amène deux conclusions. D'une part ce rapport linéaire établi entre activité et compétence ne laisse que peu de place à l'initiative humaine, comme si être compétent, c'était exclusivement répondre convenablement aux exigences de la tâche et non transformer la tâche prescrite en tâche effective (Leplat \& Cuny, 1974, op. cit.). D’autre part, cette proximité entre activité et compétence confirme, comme nous l'avons indiqué, le rôle plus évaluateur que descriptif des compétences dans les référentiels.

$\mathrm{Ne}$ peut-on envisager, dans un référentiel, une formalisation plus aboutie de la compétence, sans doute déduite des activités, mais dans un rapport non linéaire? Comment, alors, envisager sous un jour nouveau le rôle des compétences dans les référentiels, plus descriptif qu'évaluatif (Coulet, 2011, op. cit.)?

\subsection{Le référentiel du BTS « Management des unités commerciales »}

Le référentiel du Brevet de Technicien Supérieur (BTS) «Management des unités commerciales » va servir de support à notre réflexion suivante. Le diplôme de BTS, de niveau III ${ }^{7}$ possède une singularité dans le champ des diplômes professionnels. Situé à un niveau supérieur au baccalauréat, il relève donc de la réglementation des diplômes de l'enseignement supérieur et sa gestion est assurée par la direction générale pour l'enseignement supérieur et l'insertion professionnelle (DGESIP) du département ministériel en charge de l'enseignement supérieur. Cependant, pour des raisons historiques, la conception des BTS est confiée, comme les autres diplômes professionnels de niveaux V (CAP) et IV (baccalauréat professionnel), à des groupes paritaires gérés par la Direction Générale de l'Enseignement Scolaire (DGESCO) du Ministère de l'Éducation Nationale.

Cette singularité de gouvernance administrative, si elle se gère de manière plutôt harmonieuse entre services, a pourtant une influence sur l'activité des rédacteurs des référentiels de BTS. En effet, la doctrine de conception des diplômes professionnels de l'Éducation Nationale (séparation entre certification et formation) comme la méthodologie (construction successive d'un référentiel d'activités professionnelles, puis d'un référentiel de certification et absence de référentiel de formation) ne sont pas celles retenues par l'enseignement supérieur où le contenu de la formation, organisé en unité d'enseignement, est aussi l'unité de référence de la certification ${ }^{8}$, avec l'attribution des crédits européens - ECTS. En simplifiant volontairement le propos pour le rendre démonstratif, on peut évoquer des enjeux pédagogiques prioritaires d'apprentissage (de contenu, de savoirs) du côté de l'enseignement supérieur, la professionnalité du formé émergeant de la maîtrise de ces savoirs. Pour l'Éducation Nationale, l'objectif pédagogique central est le développement de l'apprenant (tout statut confondu) vers une maîtrise professionnelle.

51 Dans cet exemple, on peut s'interroger sur la fonction des référentiels. Sont-ils pensés comme des instruments au service d'un projet d'apprentissage (de savoirs, de compétences, d'un métier) ou de développement (de l'individu, du professionnel) ? Audelà de la question théorique qui articule plus ou moins ces deux processus (Yvon \& Clot, 2003), ce point oriente l'action des formateurs et enseignants et influence la conception des référentiels. 
Dans son écriture, le référentiel du BTS « Management des unités commerciales » retient des traces de cet arbitrage, comme le montrent les deux extraits de son référentiel de certification ci-dessous :

Figure 6 : Extrait du référentiel de certification du BTS « management des unités commerciales » (compétence $\mathrm{C} 1$ ).

Figure 6: Extract from the frame of reference for BTS certification in "management of commercial units" (skill C1)

Dans le cadre

Cl - MANAGER UNE ÉQUIPE COMMERCIALE

- d'un contexte professionnel donn

de la politique commerciale,

- de la politique des ressources humaines de l'entreprise

\begin{tabular}{|c|c|c|}
\hline $\begin{array}{l}\text { SAVOIR-FAIRE: } \\
\text { « Etre capable de ....* }\end{array}$ & $\begin{array}{l}\text { CONDITIONS DE REALISATION : } \\
\text { \& On domne..... }\end{array}$ & $\begin{array}{l}\text { CRITERES d'EVALUATION : } \\
\text { "On exige...... }\end{array}$ \\
\hline $\begin{array}{l}\text { C11-Constituer une équipe commerciale profession- } \\
\text { nelle et inotivé }\end{array}$ & $\begin{array}{l}\text { Dans le cadre } \\
\text { - de la réalisation de tout ou partie d'un } \\
\text { diagnostic des besoins en ressources } \\
\text { humaines } \\
\text { - d'opérations ponctuelles de recrute- } \\
\text { ment ou d'intégration de nouveaux } \\
\text { personnels } \\
\text { - de la mise en cenrre ou de la concep- } \\
\text { tion d'actions de formation. } \\
\text { - de la mise en - evurve d'actions ponc- } \\
\text { tuelles de motivation ou de stimulation }\end{array}$ & \\
\hline $\begin{array}{l}\text { 111- Déterminer les besoins en ressources humaines de } \\
\text { l'unité commerciale }\end{array}$ & $\begin{array}{l}\text { Avec } \\
\text { - les tableaux de bord commerciaux } \\
\text { - les perfoniances de l'entreprise (ama- } \\
\text { lyse des ventes) } \\
\text { - les informations concemant le person- } \\
\text { nel } \\
\text { - la reglementation du travail } \\
\text { - la politique de gestion des ressources } \\
\text { humaines de l'entreprise }\end{array}$ & $\begin{array}{l}\text { - Une évaluation des performances et des compé- } \\
\text { tences au plan individuel et collectif est réalisée } \\
\text { méthodiquement } \\
\text { - Les profils de poste sont définis en adéquation } \\
\text { avec les besoins détectés } \\
\text { - Le diagnostic aboutit a ane préconisation argu- } \\
\text { mentée d'a attions de recrutement, de formation, } \\
\text { d'animation, de stimulation et de restructuration }\end{array}$ \\
\hline
\end{tabular}

Figure 7 : Extrait du référentiel de certification du BTS « management des unités commerciales » (savoir associé S5) même remarque : illisible.

Figure 7: Extract from the frame of reference for BTS certification in "management of commercial units" (associated knowledge S5)

\begin{tabular}{|c|c|}
\hline \multicolumn{2}{|c|}{ S5 - MANAGEMENT DES UNITÉS COMMIERCLALES } \\
\hline SAVOIRS & LIMIIES DE CONNAISSANCES \\
\hline S51 - Les fondements du mangegment & $\begin{array}{l}\text { Il s'agit d' un cadre général : se limiter aux grands } \\
\text { principes. }\end{array}$ \\
\hline $\begin{array}{l}\text { 511-L'organisation } \\
\text { : notion d'organisation } \\
\text { modeles organisationnels }\end{array}$ & $\begin{array}{l}\text { Préenenter l'organisation en tant que cadre géééral de } \\
\text { toute action managériale : analyse de sa composition } \\
\text { et de son comportement } \\
\text { Présenter les principaux courants de pensée et leurs } \\
\text { apports. }\end{array}$ \\
\hline 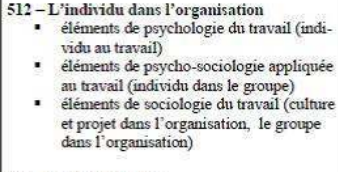 & $\begin{array}{l}\text { Définir les notions de personnalite, de motivations, } \\
\text { attitudes comportement et les criteres mesurant la } \\
\text { satisfaction de l'individu au travail. } \\
\text { Se limiter aux motivations, attitudes et componte- } \\
\text { ment de l' 'individud dans le groupe. } \\
\text { Situer l'organisation dans son environnement social. } \\
\text { Se limiter aux principaux éléments de la culture or- } \\
\text { ganisationnelle (valeurs, rituels, symboles, mythes) }\end{array}$ \\
\hline $\begin{array}{l}\text { 513 - Le management } \\
\text { : définition et enjeux } \\
\text { : métrodes de management } \\
\text { prise de décision et exercice du pouvoir }\end{array}$ & $\begin{array}{l}\text { Situer le management dans le cadre de la démarche } \\
\text { qualité. } \\
\text { Presenter le processus décisionnel et les differentes } \\
\text { théories du leadership ou styles de management. }\end{array}$ \\
\hline S52-Le manageur de l'muité commerciale & 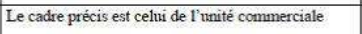 \\
\hline $\begin{array}{l}\text { 521 Les missions du manageur } \\
\text { - organisation et coordination de l'unité } \\
\text { commerciale } \\
\text { veille managériale } \\
\text { - information, animation, mobilisation et } \\
\text { - cocompagnement de l'équipe } \\
\text { controle et évaluation des performances } \\
\text { individuelles et collectives et de I'UC }\end{array}$ & 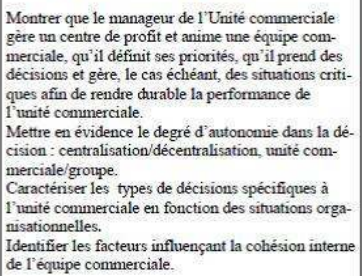 \\
\hline $\begin{array}{l}522 \text { Les outils du manageur } \\
\text { indicateurs sociaux } \\
\text { : tableaux de bord commerciaux } \\
\text { budgets } \\
\text { outilis d'aide à la décision }\end{array}$ & $\begin{array}{l}\text { Présenter les indicateurs sociaux et de gestion utiles } \\
\text { au manageur pour piloter l'unite commerciale. } \\
\text { Présenter les principaux outilis d'aide a la décision. } \\
\text { Aborder en particulier la gestion du temps person- } \\
\text { nel. }\end{array}$ \\
\hline
\end{tabular}


53 La juxtaposition dans un même référentiel de certification de compétences rédigées sous forme d'objectifs à atteindre (être capable de...) et de "savoirs associés» qui correspondent réellement à une organisation du cursus pédagogique (y compris par des consignes pour les enseignants et formateurs dans la colonne «limites de connaissances »), confirme que les rédacteurs de ce référentiel ont dû gérer le dilemme pédagogique exposé, sans pouvoir le régler dans un sens ou dans l'autre, d'où cette rédaction « hybride », certificative mais « colorée » de pédagogique.

54 Ne peut-on imaginer un système rédactionnel qui, soit accepte clairement le caractère hybride (pédagogique et certificatif) du référentiel de certification, soit assume pleinement de restreindre la rédaction et de ne retenir aucune référence à la formation?

\subsection{Le référentiel du DESJEPS « performance sportive »: généralisation ou propriétés génériques?}

Le Diplôme d'État Supérieur de la Jeunesse, de l'Éducation Populaire et du Sport (DESJEPS) spécialité « performance sportive » est un diplôme de niveau II délivré par le Ministère de la Jeunesse et des Sports. Il certifie les compétences des entraineurs sportifs.

Son référentiel de certification est construit sous forme d'unités capitalisables (UC) exprimées par un objectif principal (appelé objectif terminal d'intégration - Savy, 2013) décliné en objectifs intermédiaires (ici de premier et de deuxième rang).

Dans cet exemple, on est au cœur d'un problème fondamental que rencontre chaque concepteur de référentiel. L'objectif est de produire une description à portée générale, à partir d'une analyse d'activité professionnelle par nature singulière. Rester dans le singulier favorise la proximité au réel et la richesse des détails mais gêne la généralisation. Tendre vers le général élimine les aspérités du travail réel et fait courir le risque de l'inauthenticité. On peut alors proposer de distinguer le général du générique, comme nous avons pu précédemment le préciser :

«il faut parvenir à trouver un objet à caractère générique, c'est-à-dire qui appartient au genre, le définit, le constitue mais dont l'application ne soit pas générale, entendue comme contraire au particulier. Il faut identifier un objet spécifique, qui caractérise le métier décrit, mais qui intègre les expressions singulières » (Balas, 2011a, op. cit., p. 97). illustre la difficulté pour les rédacteurs à arbitrer entre des modes rédactionnels orientés vers le singulier ou vers le général. 
Figure 8 : Extrait du référentiel de certification du DESJEPS « performance sportive » (Unité Capitalisable $\mathrm{n}^{\circ} 1$ ).

Figure 8: Extract from the frame of reference for DESJEPS certification in "sports performance" (Capitalizable Unit $n^{\circ}$ 1)

\section{UC 1 EC de construire la stratégie d'une organisation du secteur}

OI 11 EC de préparer la prise de décision

OI 111 EC d'organiser une veille en termes d'information dans le champ d'intervention de l'organisation dans le cadre de réseaux professionnels.

OI 112 EC d'analyser les caractéristiques économiques, sociales, politiques et culturelles du territoire de référence.

OI 113 EC d'analyser l'impact des politiques publiques sur l'activité de l'organisation.

OI 114 EC de formaliser des diagnostics stratégiques pour les élus de l'organisation.

OI 12 EC d'élaborer un projet de développement

OI 121 EC de veiller au respect des objectifs, des valeurs et des méthodes de l'organisation dans une perspective éducative

OI 122 EC de mobiliser les ressources internes dans la préparation d'un projet de développement.

OI 123 EC d'animer une démarche d'ingénierie de projet.

OI 124 EC de formaliser un projet de développement.

OI 125 EC d'élaborer les dossiers de financement.

OI 13 EC de mobiliser les instances dirigeantes élues

OI 131 EC de transmettre l'information nécessaire à la prise de décision.

OI 132 EC d'exposer les alternatives stratégiques aux instances dirigeantes.

OI 133 EC de préparer les travaux des assemblées décisionnelles.

Figure 9 : Extrait du référentiel de certification du DESJEPS « performance sportive » (Unité Capitalisable $n^{\circ} 4$ ).

Figure 9: Extract from the frame of reference for DESJEPS certification in "sports performance" (Capitalizable Unit $n^{\circ} 4$ )

UC \& EC d'encadrer la discipline sportive en sécurité

OI 41 EC de réaliser en sécurité les démonstrations techniques

OI 411 EC d'évaluer les risques objectifs liés à la pratique de la discipline.

OI 412 EC d'évaluer ses propres capacités à effectuer une démonstration technique.

OI 413 EC d'expliciter les différents éléments de la démonstration technique.

OI 42 EC de réaliser les gestes professionnels nécessaires à la sécurité des pratiquants

OI 421 EC d'évaluer les risques objectifs liés à l'activité pour le pratiquant.

OI 422 EC d'anticiper les risques potentiels pour le pratiquant.

OI 423 EC de maîtriser le comportement et les gestes à réaliser en cas d'incident ou d'accident.

OI 43 EC d'assurer la sécurité des pratiquants et des tiers

OI 431 EC d'évaluer les risques objectifs liés au contexte de pratique.

OI 432 EC d'anticiper les risques juridiques liés à la pratique et au milieu dans lequel il se pratique.

OI 433 EC d'assurer la sécurité passive des équipements.

59 Pensé pour être décliné dans un second temps par discipline sportive (football, spéléologie, natation sportive), ce référentiel est porteur d'un degré de généricité, cependant bien différent entre l'UC 1 et l'UC 4. À la lecture de cette dernière, le lecteur identifie immédiatement le métier décrit : un métier d'encadrement pédagogique dans le champ du sport. À l'opposé, le même lecteur pourra difficilement identifier le métier visé par l'UC 1: s'agit-il d'un manageur de structure commerciale (voir BTS précédemment présenté) ? D'un responsable de projet travaillant seul (type consultant)?

La rédaction de cette unité renvoie à l'obstacle classique que doivent affronter les rédacteurs de référentiel : à force de vouloir rédiger un cadre permettant d'embrasser la 
diversité des situations ${ }^{9}$, le niveau de généralité de la rédaction fait, là aussi, prendre le risque d'une perte de sens.

61 Comment affronter cet exercice de la référentialisation sans perdre de vue la nature holiste de la «matière» décrite (le métier, l'activité, les compétences) ? Comment produire des référentiels favorables à une mise en mouvement et non réducteurs de dynamique ? Comment rédiger des documents de référence qui ne s'éloignent pas trop du travail réel?

\subsection{Conception d'un référentiel expérimental avec des masseurs- kinésithérapeutes : quand le "non-réalisé » est caractéristique du réel ${ }^{10}$}

Pour répondre à ces questions, nous avons voulu expérimenter une nouvelle manière de concevoir des référentiels (Balas, 2011a, op. cit.) en cherchant à développer l'expérience des professionnels associés à notre démarche. Cette nouvelle façon de penser les référentiels, inscrite dans une approche historico-culturelle (Vygotski, 2014, op. cit.), était assez « contre-intuitive » au départ pour les professionnels de la kinésithérapie associés à notre démarche. En effet, face à une prédisposition à chercher à décrire leur métier à partir de l'identification des bonnes (et en creux des mauvaises) pratiques, comme c'est devenu une tradition dans les métiers de la santé (Demailly, 2013), nous avons proposé à ces professionnels une solution différente et surprenante pour eux: identifier les dilemmes de leur métier (Prot, 2014). Nous soutenions que ces dilemmes sont des situations caractéristiques d'un métier, proches du réel, dans la mesure où elles obligent les professionnels à disposer de plusieurs réponses pertinentes possibles, afin justement de gérer, dans l'action, les contradictions situées.

Dans une situation de soin, par exemple, le professionnel doit poursuivre deux objectifs en partie non-compatibles : "soigner le patient ", c'est-à-dire appliquer des techniques de soin (massage, mobilisation...) mais aussi, dans l'espoir de le rendre autonome, " éduquer le patient à se soigner ", c'est-à-dire lui fournir les repères lui permettant de se prendre en charge, seul. Selon la situation, la nature de la pathologie, les modes relationnels établis et de très nombreux autres facteurs qui définissent les caractéristiques de la situation, l'action du professionnel sera plus ou moins orientée vers l'un ou l'autre de ces objectifs. C'est cette capacité à arbitrer qui constitue, à notre sens, un élément caractéristique de la professionnalité du soignant et qui doit donc être intégré au référentiel de son métier. Alors, on peut, en identifiant les dilemmes les plus repérables du métier, produire une description pertinente de ce dernier.

Dans cet exemple expérimenté au cours d'une recherche doctorale avec plusieurs groupes de masseurs-kinésithérapeutes, nous avons tenté de dépasser les contradictions identifiées précédemment lors de la conception de référentiel. Ici, les notions mobilisées sont moins complexes et le terme de compétence est par exemple écarté car trop polysémique. Le lien entre fonction descriptive, formative puis certificative du référentiel est précisé : ce qui est caractéristique de l'exercice du métier (l'affrontement d'un dilemme de métier) est aussi le support de construction des situations d'apprentissage (par exemple en reconstituant une situation de confrontation à un dilemme). De même, la capacité de gérer ces dilemmes devient aussi un critère d'évaluation. 

exemplaire dans la mesure où elle n'est pas reproductible, forcément, dans toutes les situations : l'identification des dilemmes d'un métier est le résultat d'une intervention en coanalyste de l'activité, avec des collectifs de professionnels. On peut envisager que l'adoption de cette "méthode ", comme entrée dans l'intervention, ne trouve pas d'écho chez d'autres groupes de professionnels. Cette expérience est plutôt là pour inviter les concepteurs de référentiels à penser autrement leur travail, au profit d'une clarification de cette fonction de conception mais favorable, aussi, à un usage « développemental » de l'instrument produit (Balas, 2011a, op.cit.). Les référentiels, en effet, peuvent, à l'usage, servir de norme " pétrifiée » (Clot, 2003) ou au contraire constituer un cadre favorable à une relance des dialogues entre professionnels sur leur métier.

\section{Portée de l'analyse et perspectives}

L'ultime proposition que nous formulons repose sur le constat que les processus de conception de référentiels sont rarement aisés et que les rédacteurs sont peu aidés par les différents guides qui tentent de normer leurs pratiques mais leur font vivre de sérieuses contradictions.

67 Ces pratiques de conception remplies de contradictions laissent des traces, dans les référentiels produits. C'est en partant de ce pari que nous avons construit un cadre méthodologique ad hoc, basé sur une lecture attentive des référentiels et sur une confrontation des rédactions, à partir d'une importante expérience de ces processus.

Ce cadre, cependant, relève du «bricolage ». Si notre conviction est que la recherche est affaire d'invention, d'activité singulière et de contournement des obstacles du réel comme tout travail, il est aussi important de s'appuyer sur des acquis de la communauté scientifique. Si nous nous sommes inspiré assez souvent des manières de faire de Fabienne Maillard (par exemple 2003; 2012) en particulier dans la lecture des référentiels, ce cadre est cependant encore fragile et mérite d'être confronté et plus clairement explicité, ce qui constitue un chantier heuristique et une limite de notre présent exposé.

On peut aussi discuter de la nature indirecte des traces obtenues sur l'activité des concepteurs. Si nous avons eu l'occasion d'observer des concepteurs en action, il n'a pas encore été possible de construire une véritable intervention visant à analyser, avec ces concepteurs, leurs activités de conception. Pour cela, il est nécessaire d'élaborer, avec eux, un véritable objectif de co-analyse, où les acteurs apportent l'expertise de leur activité face à l'expertise d'intervention du chercheur.

\section{Conclusion}

L'objet poursuivi dans ce texte peut se résumer en quelques mots: montrer que la conception des référentiels, telle qu'elle est conduite aujourd'hui en prenant peu en compte le travail réel, met ses acteurs dans des situations complexes. Les contradictions vécues, entre volonté de rendre justice au métier qu'ils souhaitent décrire et cadres méthodologique et institutionnel imposés, laissent des traces dans les documents produits. Ce sont ces traces que nous avons tenté de saisir, comme indices indirects de cette activité contrariée, à partir d'exemples de référentiels de diplômes divers, de 
secteurs et de niveaux différents. S'il est vrai que toute activité comporte des contradictions, celles vécues par les concepteurs de référentiels sont parfois paralysantes pour leur action. Ces concepteurs sont parfois dans l'empêchement à développer leur activité ou simplement à faire du bon travail...

Ces études confirment l'intérêt de repenser ces dispositifs de référentialisation en associant plus directement des professionnels des métiers concernés et en conduisant une réflexion plus poussée sur ce qui est caractéristique de l'exercice de ce métier. Doit-on répertorier les tâches? Les gestes de métier? Nous proposons de référentialiser des « irrésolus » de ce métier, afin de provoquer, artificiellement, un développement de ces activités (Balas, 2013) et du pouvoir d'agir des acteurs concernés (Clot, 2008a, op. cit.). En effet, ce type d'expérimentation montre qu'un référentiel ainsi produit est un instrument de reprise des dialogues sur le travail et non, comme actuellement, un document uniquement normatif.

Mais comment aller plus loin? Il semble qu'une analyse plus poussée de l'activité des concepteurs de diplômes soit nécessaire.

Si ce « désir d'analyse » parvient à émerger, il convient alors de disposer de conditions techniques minimum. Il faut de la disponibilité des acteurs, un cadre méthodologique adapté (par exemple l'instruction au sosie - Oddone, Ré, \& Briante, 1981), permettant de recueillir des traces, principalement langagières, de ces activités de conception. Aujourd'hui, ces conditions sont difficiles à mettre en place, en particulier parce que les rédacteurs de ces référentiels de diplômes ne réalisent ces activités que ponctuellement. Au-delà de ce travail de conception, ils sont professionnels du secteur concerné (dans nos exemples, l'aéronautique, la kinésithérapie, la gestion des unités commerciales, etc.), enseignants, inspecteurs et ne consacrent à la conception des diplômes qu'une part parfois très faible de leur temps.

De plus, les institutions qui organisent ces groupes de travail et ces CPC, ne se fixent pas pour priorité d'analyser l'activité de conception des référentiels de leurs diplômes, mais s'intéressent plutôt aux produits finis et à leur congruence et à leur « acceptabilité » par les systèmes de formation qui les mettent en œuvre.

Il nous semble, pourtant, qu'un approfondissement de ce travail, à partir d'une intervention en analyse du travail pourrait participer à mieux comprendre ces processus complexes et à construire des repères pour les acteurs.

76 Cette proposition est d'autant plus importante que des travaux montrent qu'il existe un lien étroit entre «la norme et l'usage» (Maillard, 2001, op.cit.). En effet, une norme construite sur la base d'une analyse détaillée du travail réel permet dans un second temps un usage plus riche, par exemple pour les enseignants et formateurs en charge de mettre en œuvre le diplôme professionnel visé.

On pourrait même allez plus loin et envisager, comme ce fut le cas dans une intervention passée avec des masseurs-kinésithérapeutes (Balas, 2011a, op.cit.) ou plus récente avec des manageurs (Balas, Bourgeois, Castel, \& Théry, 2015) de considérer les référentiels comme des normes à entretenir régulièrement et ainsi en faire des "prétextes " à la relance régulière de dialogues au sein des groupes professionnels concernés. 


\section{BIBLIOGRAPHIE}

Akrich, M. (1991). L'analyse socio-technique. In D. Vinck (Ed.), La gestion de la recherche, (pp. 339-353). Bruxelles : De Boeck.

Albero, B. (2010). Une approche sociotechnique des environnements de formation. Rationalités, modèles et principes d'action. Éducation et Didactique, 4(1), 7-24.

Albero, B., \& Brassac, C. (2013). Une approche praxéologique de la connaissance dans le domaine de la formation. Éléments pour un cadre théorique. Revue française de pédagogie, $\mathrm{n}^{\circ}$ 184, 105-120

Balas, S. (2011a). Le référentiel, un outil de formation, un instrument de développement du métier. Le métier de masseur-kinésithérapeute en référence. Thèse de doctorat de sciences de l'éducation. Paris: CNAM, http://tel.archives-ouvertes.fr/tel-00669690

Balas, S. (2011b). Kinésithérapeute, un métier de référence. Nouvelle revue de psychosociologie, no $12,2011 / 2,223-238$.

Balas, S. (2013). Intervenir avec des masseurs-kinésithérapeutes : le développement en référence. Activités, 10(1), 93-111, https://activites.revues.org/584

Balas, S. (2014). Le référentiel : un instrument pour « manœuvrer les marges » ? Communication dans le cadre de la $14^{\text {ème }}$ journée internationale de sociologie du travail. Les marges du travail et de l'emploi : formes, enjeux, processus, Lille, 17-19 juin 2014.

Balas, S., Bourgeois, F., Castel, D., \& Théry, L. (2015). L'analyse du travail comme objet et comme moyen d'une «formation-action » des manageurs. Symposium dans le cadre du $50^{\text {ème }}$ congrès de la SELF, «Articulation performance et santé dans l'évolution des systèmes de production », Paris, 23, 24, 25 septembre 2015

Balas, S., Paddeu, J., \& Veneau, P. (2016). Évaluation certificative dans les diplômes professionnels : facteur humain et équité. Éducation Permanente, hors-série AFPA, 45-51

Boccara, V., Vidal-Gomel, C., Rogalski, J., \& Delhomme, P. (2014). Concevoir des référentiels comme des outils de formation? Réflexions à partir de la formation initiale à la conduite automobile. In B. Prot (Ed.), Les référentiels contre l'activité. En formation, gestion, certification (pp. 119-132). Toulouse : Octarès.

Briec, C. (2013). Syndicalisme : « l'impersonnel » à l'épreuve. Le cas d'une section syndicale du SNUIPP-FSU. Thèse de doctorat en psychologie du travail. Paris : CNAM.

Canguilhem, G. (2002). Écrits sur la médecine. Paris : Le Seuil

Champy-Remoussenard, P. (2005). Les théories de l'activité entre travail et formation. Savoirs, 2005/2, no 8, 9-50

Chauvigné, C. (2010). Les référentiels en formation. In C. Chauvigné \& Y. Lenoir (Eds.). Les référentiels en formation : enjeux, légitimité, contenu et usage, Recherche \& Formation, $\mathrm{N}^{\circ} 64-2010$, 77-89

Clot, Y. (1999). La fonction psychologique du travail. Paris : PUF.

Clot, Y. (2000). La formation par l'analyse du travail : pour une troisième voie. In B. Maggi (Ed.) (2000). Manières de penser, manières d'agir en éducation et en formation (pp. 133-156). Paris : PUF. 
Clot, Y. (2003). Le collectif dans l'individu? Actes du $38^{e}$ colloque de la SELF, Modèles et pratiques de l'analyse du travail 1988-2003, 15 ans d'évolution, Paris, www.ergonomie-self.org.

Clot, Y. (2004). Action et connaissance en clinique de l'activité. Activités. [en ligne], 1(1), https:// activites.revues.org/1145

Clot, Y. (2008a). Travail et pouvoir d'agir. Paris : PUF

Clot, Y. (2008b). La recherche fondamentale de terrain : une troisième voie. Éducation Permanente, $177,2008 / 4$.

Coulet, J.-C. (2011). La notion de compétence : un modèle pour décrire, évaluer et développer les compétences. Le Travail Humain, 74, 2011/1, 1-30

Demailly, L. (2013). Le nouveau management public et les particularités des secteurs d'action publique : le cas de la santé en France. Éducation et sociétés, 2013/2, nº 32, 75-91.

Fernandes, J., \& Santos, M. (2014). L'activité des formateurs dans le cadre de la reconnaissance et la validation des acquis : l'évolution d'un instrument à partir de l'activité. In numéro spécial de la revue électronique Pistes : L'analyse ergonomique du travail et de la formation : quelques contributions récentes, 16(4), 2014

Figari, G. (1994). Évaluer : quel référentiel ? Bruxelles : De Boeck Université, Guide d'élaboration des diplômes professionnels, CPC Documents, 2004-7

Hoc, J.-M. (1996). Supervision et contrôle de processus. La cognition en situation dynamique. Grenoble : PUG.

Huchette, M. (2014). Stages en entreprise : quelles références pour les enseignants des disciplines technologiques? In B. Prot (Ed.) Les référentiels contre l'activité. En formation, gestion, certification. (pp. 71-87). Toulouse : Octarès,

Jobert, G. (2013). Le formateur d'adultes : un agent de développement. Nouvelle revue de psychosociologie, 2013/1, 15, 31-44.

Jouvenot, C., \& Parlier, M. (Sous la dir.), (2005). Élaborer des référentiels de compétences : principes et méthodes. Lyon : ANACT Editions

Lainé, A. (2011). Répondre de son expérience par le récit de soi, Éducation Permanente, no 187 , 2011/2, 81-93.

Liaroutzos, O., \& Sulzer, E. (2006). La méthode ETED. De l'analyse du travail aux référentiels d'emploi/métier. Relief, Echanges du Céreq, 14, février 2006

Leontiev, A. N. (1984). Activité, conscience, personnalité. Moscou : Éditions du Progrès.

Leplat, J. (2002). Psychologie de la formation. Jalons et perspectives. Toulouse : Octarès

Leplat, J., \& Cuny, X. (1974). Les accidents du travail. Paris: PUF

Leplat, J., \& Hoc, J.-M. (1983). Tâche et activité dans l'analyse psychologique des situations. Cahiers de psychologie cognitive, 3(1), 49-63

Maggi, B. (2011). Interpréter l'agir : un défi théorique. Paris : PUF, Collection le travail humain.

Maillard, F. (2001). Les référentiels des diplômes professionnels : la norme et l'usage. Revue des commissions professionnelles consultatives, 2001-5

Maillard, F. (2003). Les référentiels des diplômes professionnels confrontés à leurs critiques : une mise en valeur de leurs enjeux sociaux. Revue française de pédagogie, ํํ145, octobre-décembre 2003, 63-76 
Maillard, F. (2007). Les diplômes professionnels de l'éducation nationale dans l'univers des « certifications professionnelles » : une singularité mise en question, L'orientation scolaire et professionnelle [En ligne], 36/2 | 2007, mis en ligne le 01 juin 2010, consulté le 26 août 2010. http://osp.revues.org/index1348.html

Maillard, F. (dir.) (2012). Former, certifier, insérer. Effets et paradoxes de l'injonction à la professionnalisation des diplômes. Rennes : Presses Universitaires de Rennes.

Mandon, N. (2009). Analyser le sens et la complexité du travail, la méthode ETED (Emplois Types en Dynamique). Paris : L'Harmattan, collection « Action et savoirs clés ».

Marcel, J-.F., \& Savy, H. (2013). Évaluons, évoluons. L'enseignement agricole en action. Dijon : Educagri éditions

Mayen, P., \& Métral, J.-F. (2008). Compétences et validation des acquis de l'expérience. Formation emploi, nº 101, 2008, 183-197.

Oddone, I., Ré, A., \& Briante, G. (1981). Redécouvrir l'expérience ouvrière. Vers une autre psychologie du travail ? Paris : Éditions sociales

Olry, P., \& Vidal-Gomel, C. (2011). Conception de formation professionnelle continue : tensions croisées et apports de l'ergonomie, de la didactique professionnelle et des pratiques d'ingénierie. Activités, 8(2), 115-149, https://activites.revues.org/2604

Poussin, N. (2010). Répliquer collectivement à la tâche pour « faire du bon boulot » d'un « boulot sale ». Travailler, 2010/2 no 24, 93-110.

Prot, B. (2003b). Analyse du travail des jurys en validation des acquis : l'usage du référentiel. Revue de l'orientation scolaire et professionnelle, 32/2, 219-243.

Prot, B. (2014). Les référentiels contre l'activité - En formation, gestion, certification. Toulouse : Éditions Octarès

Savy, H. (2013). L'enseignement agricole, un système de formation original. In. C. Szymankiewicz (Ed.), Les Notices : Le système éducatif en France (pp. 207-222). Paris : La documentation française.

Schwartz, Y. (2007). Un bref aperçu de l'histoire culturelle du concept d'activité. Activités, 4(2), 122-133, https://activites.revues.org/1728

Szymankiewicz, C. (Ed.). (2013, $4^{\text {ème }}$ édit.). Les Notices : Le système éducatif en France. Paris : La documentation française.

Ughetto, P. (2014). Référentiels de compétences : ce que l'instrument fait à a logique compétence. In B. Prot (Ed.), Les référentiels contre l'activité (pp. 35-49).Toulouse : Octarès.

Vygotski, L. (1925/2005, traduction de Sève, F.). Psychologie de l'art. Paris : La Dispute.

Vygotski, L. (1934/1997, traduction Sève F.). Pensée et langage. Paris : La Dispute.

Vygotski, L. (2014). Histoire du développement des fonctions psychiques supérieures. Paris : La Dispute.

Yvon, F., \& Clot, Y. (2003). Apprentissage et développement dans l'analyse du travail enseignant. Pratiques psychologiques, 2003-1, 19-35. 


\section{NOTES}

1. Sur la complexité de la prescription, dimension « impersonnelle » du métier, voir la thèse de Briec (2013).

2. Par exemple dans le champ de la santé ou de la scolarité

3. Le nom du diplôme joue ici un grand rôle. Certains intitulés sont abscons (comme par exemple le BTS «Fluides, Energies, Environnement ») alors que d'autres sont plus accessibles (comme le récent bac pro "facteurs d'orgues»). On constate d'ailleurs des débats très vifs sur la dénomination des diplômes dans les commissions paritaires chargées de valider ces diplômes, les commissions professionnelles consultatives (CPC).

4. C'est-à-dire les certificateurs.

5. Cet extrait de référentiel, comme les suivants présentés dans ce texte, est identique dans sa forme publiée.

6. La plupart des certificateurs (éducation nationale, agriculture) ne traitent pas des questions de formation dans les référentiels, afin de marquer, dans un système "tiré par l'évaluation ", la distinction entre formation et certification (Marcel \& Savy, 2013) mais aussi pour offrir un espace d'initiative aux équipes pédagogiques. Cependant, le champ de la santé ne suit pas cette tendance en proposant des référentiels de formation détaillés, comme l'illustre le diplôme d'Etat rénové d'infirmier qui comprend un référentiel de formation très complet.

7. En France, la nomenclature des niveaux de formation reste celle en vigueur depuis la fin des années 60 qui prévoit cinq niveaux (du V vers le I) en référence, essentiellement, à une durée du cursus d'études.

8. Même si les récentes adoptions législatives vont conduire à imposer la rédaction d'un référentiel de compétences pour chaque diplôme de l'enseignement supérieur (Szymankiewicz, 2013)

9. Et c'était le cas ici, puisque les unités $\mathrm{n}^{\circ} 1$ et 2 étaient communes avec l'autre spécialité du DESJEPS intitulée « animation socio-éducative ou culturelle ».

10. Ce sous-titre fait référence aux travaux de Clot (1999) qui distingue le « réalisé du réel » dans son analyse des activités de travail, ce que l'on renonce à faire caractérisant tout autant le réel que « la solution qui a vaincu » (le réalisé).

\section{RÉSUMÉS}

L'objet de ce texte est de montrer que la conception des référentiels pour les diplômes, telle qu'elle est conduite aujourd'hui, en prenant peu en compte le travail réel, met ses acteurs dans des situations complexes. Les contradictions vécues laissent des traces dans les documents produits. Nous avons tenté de saisir ces traces à partir d'exemples de référentiels de diplômes. Une proposition alternative permet de produire un résultat utile à la réflexion mais aussi d'envisager une activité de conception plus cohérente.

The object of this text is to show that the design of frames of reference for diplomas, as it is led today, taking little account of the real work, puts the actors concerned into complex situations. The real-life contradictions leave traces in the documents produced. Using frames of reference 
for diplomas, we attempted to grasp these traces. An alternative proposition makes it possible to produce a result that is useful for future reflection, and to envisage a more coherent design process.

INDEX

Keywords : design, frame of reference, diploma, actual work Mots-clés : conception, référentiel, diplôme, travail réel

\section{AUTEUR}

\section{STÉPHANE BALAS}

19, rue Charles Landelle, 53000 Laval - stephane.balas@wanadoo.fr 